\title{
Umkämpfte Nachhaltigkeit - vergessene Leiblichkeit. Der Fall der Wildpferde in Namibia
}

\author{
Robert Pütz und Antje Schlottmann
}

\section{Zusammenfassung}

Der Beitrag analysiert Konflikte um nachhaltigen Naturschutz. Er zeigt erstens, inwiefern diese in divergierenden Vorstellungen von Natur gründen, z. B. welche Tiere lokale Ökosysteme repräsentieren (dürfen), zweitens, dass Diskursanalysen diese Konflikte nicht hinreichend erfassen, da sie eine agency of nature ausblenden (müssen), und drittens, dass Naturschutzkonflikte immer auch inkorporierte Praxis sind, wenn z. B. die verinnerlichte Normativität von Nachhaltigkeit mit der leiblichen Erfahrung der Arbeit mit Tieren in Widerspruch tritt. In den dominierenden Rationalitäten von Nachhaltigkeit findet diese Dimension keinen Platz.

\section{$1 \quad$ Einleitung}

Der Namib-Naukluft Park im Südwesten Namibias ist einer der größten Nationalparks weltweit und beheimatet einige der wichtigsten touristischen Attraktionen des Landes. Der aktuelle Management Plan verfolgt das Ziel, die ,Nachhaltigkeit und Wettbewerbsfähigkeit“ von zonierten Landnutzungseinheiten ,zu demonstrieren“ (MET 2013, S. 9). Weiterhin wird betont, dass Nachhaltigkeit zu einer der

$\overline{\text { R. Pütz }(\varangle) \cdot \text { A. Schlottmann }}$

Institut für Humangeographie der Goethe-Universität Frankfurt am Main, Frankfurt am Main, Deutschland

E-Mail: puetz@uni-frankfurt.de

A. Schlottmann

E-Mail: schlottmann@geo.uni-frankfurt.de 
Säulen des Parkmanagements zähle (ebd., S. 27). Was sich in solchen Darstellungen wie selbsterklärend liest, ist in der Praxis ein hochgradig umkämpftes Feld um die Fragen, wer oder was für wen oder was mit welcher Begründung wie zu schützen ist. Dies zeigt das Beispiel der wildlebenden Pferde im Park, die durch mehrjährige Dürren und jüngst das Vordringen von Hyänen vor dem Aussterben stehen. Was für die einen - große Teile des Parkmanagements und des namibischen Umweltministeriums - der „Lauf der Natur“ ist, erfordert für andere - NGOs und Akteure des lokalen Tourismusgewerbes - Maßnahmen des Menschen zum Schutz der Wildpferde.

Zur Untersuchung dieses Falls nehmen wir auf Basis von Feldarbeiten ${ }^{1}$ im Folgenden erstens eine diskursanalytische Perspektive ein und zeigen, wie in dem Konflikt um „nachhaltigen“ Umgang mit den Wildpferden unterschiedliche Naturvorstellungen mit Fragen von Schutzwürdigkeit verknüpft sind. Insbesondere die Frage, welche Tiere das lokale Ökosystem der Namib repräsentieren (dürfen), erweist sich als umstritten, widersprüchlich und folgenreich.

In einer zweiten Betrachtung argumentieren wir, dass die Durchsetzung von Nachhaltigkeit immer mit symbolischen und damit einhergehenden materiellen Praktiken der Grenzziehung verbunden ist. Die Territorialisierung von Naturschutz in Parks ist die Verräumlichung eines diskursiv verankerten dualistischen Verständnisses von Natur als Gegenwelt zu einem wie auch immer gestalteten kulturellen Ordnungsprinzip. Auch Territorialisierungen, das symbolische Eingrenzen wie das materielle Umzäunen, sind umkämpft. Sie können den unterschiedlichen Ansprüchen an Natur und ihrem Schutz dienen oder aber auch entgegenstehen. Darüber hinaus zeigt sich die Konflikthaftigkeit der Grenzarbeit aber insbesondere mit Blick auf die materiellen und körperlichen Widerständigkeiten der Pferde sowie ihres Lebensraumes. So wird deutlich, dass rein diskursanalytische Verfahren Konflikte nachhaltigen Naturschutzes nur unvollständig reflektieren können, da sie Aspekte einer agency of nature ausblenden (müssen). Wie wir zeigen, können sie jedoch sinnvoll in ein Konzept von Assemblage eingebettet werden, das diskursiv verhandelte Naturvorstellungen als wesentlichen, aber eben nur einen Teil eines Mensch-Wildpferd-Netzwerkes

\footnotetext{
${ }^{1}$ Interviews vor Ort mit Beamten des Ministeriums für Umwelt und Tourismus (MET), Naturparkmanagern, Aktivisten der Namibia Wild Horses Foundation (NWHF) und touristischen Anbietern, Analysen von Programmen (Strategien zum Nationalparkmanagement u. a.) sowie Auswertungen von Printmedien (Artikel und Leserbriefe in namibischer Presse) und Internetforen (Facebook). Die Interviews wurden in deutscher und englischer Sprache geführt. Eine frühere Version des vorliegenden Beitrages wurde bereits in englischer Sprache veröffentlicht (Pütz und Schlottmann 2020)
} 
begreift, das sich aus Menschen und Nicht-Menschlichem (Tiere, Technologien, Diskurse, Materialitäten) zusammensetzt.

Schließlich zeigt unser Fall, dass konfliktreiche Grenzarbeit auch als inkorporierte Praxis von Subjekten erscheint, deren verinnerlichte Normativität von Nachhaltigkeit mit ihrer leiblichen Erfahrung bei der Arbeit mit Tieren zu inneren Konflikten führt - wenn etwa Entscheidungen getroffen werden müssen, die für die Tiere Leben oder Tod bedeuten. In den dominierenden Rationalitäten von Nachhaltigkeit findet diese Dimension keinen Platz. In einem dritten Schritt prüfen wir daher mit dem Konzept der Interkorporalität eine Perspektive, die eine andere wissenschaftliche Betrachtung von Mensch-Tier-Interaktionen ermöglicht und nicht zuletzt auch einer Neuverhandlung der Tauglichkeit des Begriffs der Nachhaltigkeit dienlich sein kann.

Ausgangspunkt für unsere Annäherung an den Fall ist, dem breiten gesellschaftlichen „Konsens zu Nachhaltigkeit“ kritisch zu begegnen (Schwartz 2016) und ihn vor allem auf die Relationalität und prinzipielle Unterbestimmtheit des Begriffs zurückzuführen. „Nachhaltigkeit“ kann sich auf alles Mögliche beziehen und daher auch oftmals zuwiderlaufende Felder gesellschaftlicher Praxis wie die der Naturnutzung und des Naturschutzes scheinbar unproblematisch vereinen. Mit Laclau (2002) gesprochen ist Nachhaltigkeit ein ,leerer Signifikant“, der eher dazu dient, gesellschaftliche Kontroversen zu verhüllen, denn sie zum Vorschein $\mathrm{zu}$ bringen. Insofern scheint für die wissenschaftliche Betrachtung angezeigt, zunächst nach der Verwendung des Begriffs in der Praxis zu fragen, um dessen Bedeutung zu erkennen (Tremmel 2003, S. 63). Brand und Jochum (2000, S. 174) zufolge geht es um die Analyse eines kontrovers strukturierten Diskursfelds, ,auf dem verschiedene Akteure um die Durchsetzung ihrer spezifischen Deutung von Nachhaltigkeit und den daraus sich jeweils ergebenden unterschiedlichen Nachhaltigkeitsstrategien kämpfen“. Wir schließen an diesen pragmatischen Zugriff an und begreifen Nachhaltigkeit in ihrem unbestimmten Gehalt heuristisch als etwas, für das - auch bei Konzepten ,nachhaltiger Entwicklung“ - eine Praxis des Bewahrens bzw. Verdauerns konstitutiv ist, vor allem, wenn die ökologische Sphäre von Nachhaltigkeit angesprochen ist. Gleichzeitig erweitern wir den machtsensiblen Zugang über die diskursive Ebene hinaus und nutzen den Begriff als Zugang zu einem sich um ihn entfaltenden umkämpften Feld gesellschaftlicher Auseinandersetzung, das es in seinen Dimensionen mit Blick auf räumlich und zeitlich situierte diskursive und materielle Praktiken und deren Verhältnis zu erfassen gilt. 


\section{Fallstudie: Die Wildpferde der Namib}

\subsection{Herkunft und Geschichte}

Die namibischen Wildpferde sind eine Vermischung von Nachkommen verschiedener europäischer Pferderassen, die Ende des 19. Jahrhunderts auf unterschiedliche Weise in die Region Garub gelangten, etwa $100 \mathrm{~km}$ von Lüderitz entfernt am Rand der Namib-Wüste. Durch Diamantenfunde hatte Lüderitz einen ungeheuren Aufschwung erfahren. Zur Überwindung seiner isolierten Lage zwischen Wüste und Meer wurde eine Eisenbahnlinie nach Aus gebaut und bei Garub ein ergiebiger Grundwasserspeicher entdeckt und angezapft (vgl. Abb. 1). Bis heute bildet der damals angelegte Brunnen die Basis für die Existenz der Pferdeherden der Namib.

Die Vorfahren der heutigen Wildpferde kamen auf dreierlei Weise nach Namibia und zur Wasserstelle von Garub (vgl. Goldbeck et al. 2011): Eine erste war der Import von Zuchtstuten und -hengsten nach damals Deutsch-Südwestafrika (1884-1915). Die deutschen Kolonialisten benötigten Arbeitspferde und hielten Rennpferde zur Freizeitgestaltung der durch Diamantenhandel vermögend gewordenen Bevölkerungsteile. Für beide Zwecke wurde eine Zucht in Kubub, 35 km südöstlich von Garub, aufgebaut. Ein zweiter Ahnenzweig waren Pferde, die die deutschen „Schutztruppen“ zur Kriegsführung benötigten (vgl. Abb. 2). Insbesondere im Vorfeld des Ersten Weltkriegs stieg dieser Bedarf erheblich - historische Quellen belegen Ladungen aus Deutschland mit mehr als 4000 Pferden pro Schiff. Im Ersten Weltkrieg kämpften die deutschen Truppen gegen Verbände der mit England verbündeten Südafrikanischen Union. Deren „Kap-Pferde“, selber eine Neuzüchtung, sind der dritte Ahnenzweig der heutigen „Namib Horses“.

Insgesamt lagerten im Ersten Weltkrieg rund 8000 Pferde der Kriegsparteien bei Garub. Bei einem Luftangriff auf südafrikanische Truppen am 27.3.1915 wurden alleine 1700 von ihnen versprengt (ebd.: S. 36). Zudem hatten die nach Deutschland geflohenen Zivilisten ihre Arbeitspferde ausgesetzt, wie auch Pferde der unterlegenen deutschen Soldaten auswilderten. Drittens wurde das Gestït in Kubub aufgegeben und die dortigen Zuchtpferde zerstreuten sich in den benachbarten Bergen. Während die Pferde der Soldaten vornehmlich Wallache waren, lebten im Gestüt fortpflanzungsfähige Stuten und Hengste. Deshalb ist ihr Anteil am Genpool der heutigen Namib-Pferde am größten (ebd.: S. 38). Die in den Wirren der Kriegshandlungen auf unterschiedliche Wege freigesetzten Pferde fanden sich später in größeren Herden zusammen, die vor allem die Suche nach Wasser antrieb. Dies fanden sie in Garub im dort gebohrten Brunnen vor, was die Region in einem Umkreis von $30 \mathrm{~km}$ seither zum Lebensraum der Pferde machte. 


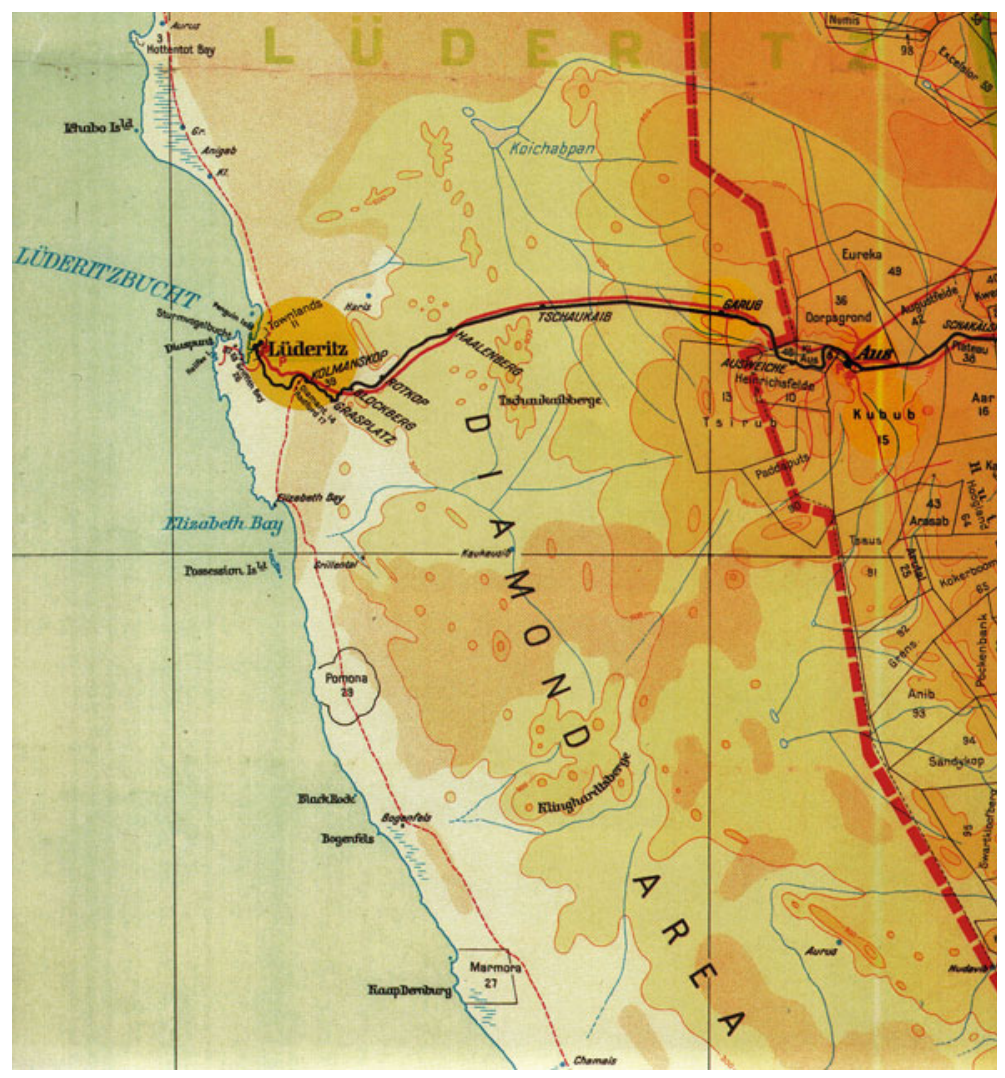

Abb. 1 Historische Karte des südlichen Teils der Namib-Wüste (Goldbeck et al. 2011, S. 26). Die Wildpferde leben um Garub, die Grenzen des Naukluft Parks durchschneiden ihren Lebensraum im Süden (entlang der Eisenbahnlinie) und Osten (östlich von Garub)

Wie konnte ein Bestand von schwankend zwischen 100 und 300 Pferden über mehr als hundert Jahre in einer lebensfeindlichen Umgebung überleben? Erstens, weil sie aufgrund der Diamantenvorkommen immer in einem Territorium mit eingeschränktem Zugang lebten. Ein streng überwachtes Betretungsverbot existierte mehr oder weniger kontinuierlich seit der Zeit der deutschen Besetzung. 1979 und 1986 wurde zudem ein Teil ihres Lebensraums dem Namib-Naukluft Nationalpark angeschlossen. Dies setzte einerseits den hohen territorialen Schutz fort - die Tiere gerieten unter Naturschutz und das Gebiet wurde eingezäunt, um sie 


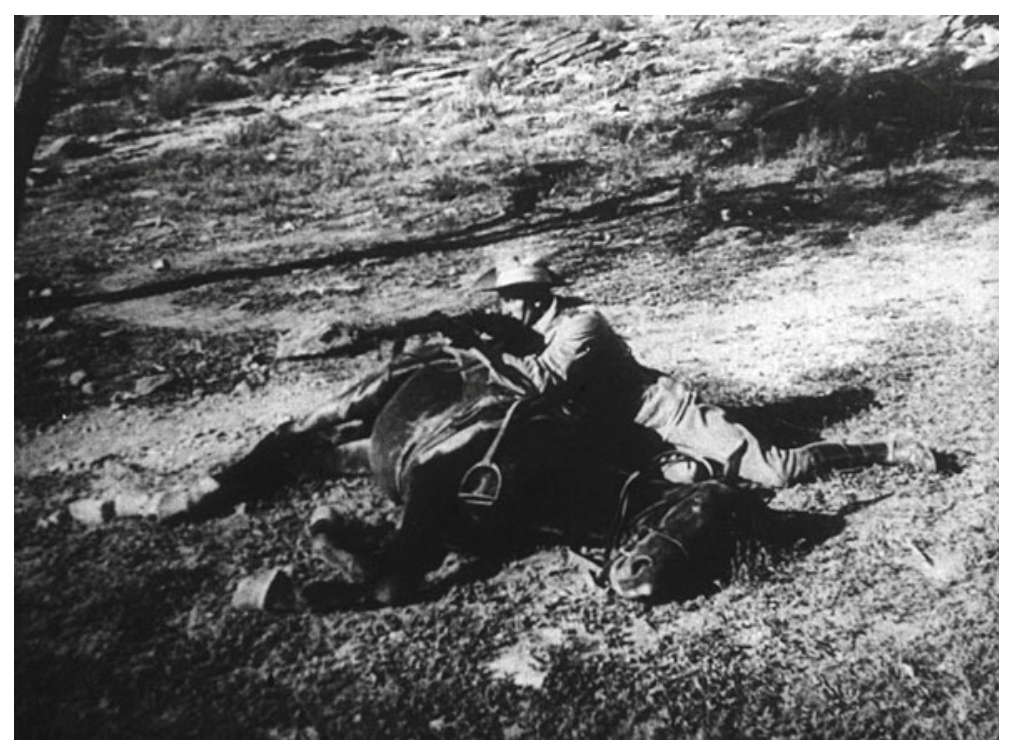

Abb. 2 Soldat der Schutztruppe hinter Pferd (Bildarchiv der Deutschen Kolonialgesellschaft, Universitätsbibliothek Frankfurt am Main, 037-0600-36). Pferde wurden im Krieg in vielerlei Funktionen als ,working animal“ eingesetzt. Hier dient es einem Soldaten als lebendiger Schutzschild und Gewehrauflage zugleich

vor Wilderern zu schützen (ebd.: S. 45) -, beschränkte andererseits aber auch die Mobilität der Pferde und fixierte deren Weidemöglichkeiten.

Zweitens war durch den Brunnen von Garub bis in die Gegenwart eine Wasserversorgung kontinuierlich gesichert. Im Krieg wurde die Bohrung zur Versorgung der einen oder anderen Kriegspartei aufrechterhalten. Später wurde sie zur Versorgung der Eisenbahnlinie gepflegt. Nach deren Umstellung auf Dieselantrieb setzte ein Sicherheitsbeamter der Diamantenfördergesellschaft CDM durch, dass die Bohrung auf Kosten der CDM nun zur Versorgung der Pferde weiter gewartet und durch Tränken mit Schwimmerventilen pferdegerecht umgestaltet wurde (ebd.: S. 43). Heute wird die Bohrung durch den Nationalpark instandgehalten.

Mit der Angliederung an den Nationalpark gerieten die Wildpferde in den Fokus einer breiteren (internationalen) Öffentlichkeit. Bis dahin war die Tatsache weitgehend unbemerkt geblieben, dass von den in günstigen Witterungsperioden bis zu 300 Pferden in Dürrezeiten viele verhungerten. Nun aber, in einer schweren Dürreperiode Ende der 1990er Jahre, wurde von Touristen und lokaler 
Bevölkerung der Anblick verendender Pferde als Problem an die Nationalparkverwaltung adressiert. Zeitgleich entstanden private Initiativen, die sich dem Schutz der Wildpferde verschrieben, allen voran die Namibia Wild Horses Foundation (NWHF), die Öffentlichkeitsarbeit betreibt, um Spendengelder für die Wildpferde zu sammeln und Druck auf Regierung und Nationalparkmanagement aufzubauen. Großen Einfluss hatte auch der weltweit ausgestrahlte Spielfilm „Running Free“ (2000), der die Geschichte der Namib-Pferde als Symbol für Freiheit stilisiert, sowie die durch ihn ausgelöste Berichterstattung in internationaler Presse und TV-Dokumentationen. Im Ergebnis wurde zugestanden, dass die Pferde in Dürrezeiten durch die NWHF Zufütterung von Heu erhalten durften, der dritte Faktor für ihr Überleben bis in die Gegenwart.

\subsection{Der aktuelle Konflikt}

In den 2010er Jahren geriet das weitgehend stabile Schutzsystem aus territorialer Isolation, gesicherter Wasserversorgung und in Dürrezeiten gesicherter Futterversorgung in die Krise. In der ersten Hälfte der 2010er Jahre war das Nahrungsangebot um Garub witterungsbedingt sehr groß gewesen, weswegen die Gegend auch von Antilopenarten (Oryxe, Springböcke u. a.) besiedelt wurde. Diesen folgten Raubtiere nach, insbesondere Hyänen. Von der NWHF wurde deren Ankunft zunächst noch begrüßt, weil sie ein Phänomen zu beseitigen schienen, das Wildpferdeherden angesichts fehlender natürlicher Feinde weltweit auszeichnet: eine starke Zunahme der Population:

„Also vor drei Jahren habe ich noch gesagt: Danke! Die Hyänen kommen rein! Wir waren mit 300 Pferden über unser carrying capacity, über der Tragfähigkeit dieses Gebietes. Und da haben wir gesagt: Super! Die kommen rein! “ (NWHF Aktivist 2017)

2014 setzte dann aber eine bis in die Gegenwart (2018) anhaltende Dürre ein, in deren Folge das Gras verdorrte und viele Tiere das Gebiet wieder verließen. Die Pferde waren hierzu jedoch nicht in der Lage. Sie waren an die Nähe der Wasserstelle gebunden und wurden durch den Zaun des Nationalparks daran gehindert, in Richtung höhergelegenes, feuchteres privates Farmland zu wandern. Der ursprünglich zum Schutz der Tiere errichtete Zaun erwies sich nun als Falle. Im Unterschied zu früheren Dürrezeiten wurden nämlich die Hyänen sesshaft: Sie folgten nicht mehr den weiterwandernden Antilopen, sondern blieben in Garub, da die Wildpferde, insbesondere die Fohlen und älteren Stuten, für sie ein leicht verfügbares Nahrungsangebot darstellten. 
In der Folge ging die Zahl der Tiere drastisch zurück und von den verbliebenen rund 100 Wildpferden lebten nur noch 33 Stuten in Garub (Swilling 2020). Gesamtzahl und Geschlechterverhältnis bedrohen die Reproduktion akut, so dass die NWHF im Januar 2017 ein faktisches Aussterben der Namib-Wildpferde bis August 2017 prognostizierte und öffentlich machte. Als Reaktion auf den medialen Druck erließ das Umweltministerium eine temporäre Genehmigung zur Fütterung der Hyänen. Auf dieser Grundlage kauft die NWHF seit 2017 auf Spendenbasis Fleisch von Wildfarmen und verfüttert es an die Hyänen. Seitdem ist der Bestand an Wildpferden weitgehend stabil.

\section{Umkämpfte ökologische Nachhaltigkeit: Welche Natur? Welche Nachhaltigkeit?}

Die Situation der akut vom Aussterben bedrohten Namib-Wildpferde erlaubt, die diskursive und materiell-praktische Umkämpftheit von Nachhaltigkeit exemplarisch zu rekonstruieren. Wie wird nachhaltiges Tun und Sein unterschiedlich gedeutet und von wem? Welche Ansprüche werden hieraus geltend gemacht und welche Widersprüchlichkeiten und Konflikte bringt dies hervor? Wie sich bereits in der kurzen Darstellung abzeichnet, treffen in der Auseinandersetzung über den ,richtigen“ Umgang mit Hyänen und Pferden fundamentale Fragen nach ihrer Zugehörigkeit zu Natur (und damit verbundenem Naturschutz) auf Fragen ökonomischer Bilanzierung und auf soziale Fragen der Verteilung von Artikulationsrechten und Zugangsmöglichkeiten zu Naturschutzgebieten. Für die Tiere steht die Bewahrung ihres Lebens und für die mit ihnen befasste Tierbiologin das Ziel des genetischen Überlebens der Wildpferde zur Disposition.

Im ersten Schritt unserer Analyse zeigen wir nun, dass für das Verständnis der Praxis ökologischer Nachhaltigkeit die Frage nach den zugrunde liegenden Naturvorstellungen zentral ist. Erst in Relation zu dem, was jeweils als Natur gilt, lässt sich verstehen, mit welchen Inhalten, Normen und Werten Nachhaltigkeit als Ziel verbunden und in materieller Praxis auch verwirklicht wird. Letzteres hat Konsequenzen für die Fragen, welche Natur für die Zukunft erhalten wird und nicht zuletzt auch, wer und was in ihr leben soll und darf. Wir folgen dabei einer Systematisierung von Mace (2014), die im Kontext Naturschutz vier Phasen gesellschaftlicher Naturkonzeptionen (framings) im Mensch-Natur-Verhältnis identifiziert. Diese können sich - wie in unserem Beispiel - zeitlich überlappen, wodurch die mit ihnen verbundenen Motive der nachhaltigen Bewahrung konflikthaft werden: Auf eine Vorstellung nature for itself, in der es primär um den Erhalt von unberührter Natur und Wildnis geht, folgt eine Phase der Frage, wie diese 
Natur trotz technischen Fortschritts und Bewirtschaftung erhalten werden kann. Mensch und Natur erscheinen dabei als separate Einheiten (nature despite people). Etwa ab der Jahrtausendwende verschiebt sich der Fokus auf Ökosysteme unter Einschluss des Menschen und die Vorstellung, aus einer Gleichzeitigkeit von Erhalt und Bewirtschaftung von Natur möglichst dauerhafte Vorteile für den Menschen zu erzielen (nature for people). Für die letzten 10 Jahre konstatiert Mace eine Tendenz, von diesem utilitaristischen Schutzgedanken abzurücken und die Wechselseitigkeit der dynamischen Beziehung zwischen Natur und Mensch anzuerkennen (nature and people).

Naturvorstellungen korrespondieren mit Vorstellungen von Wildnis, die stärker räumlich gefasst sind. Dabei spielen neben bestimmten Pflanzenarten bestimmte Tierarten eine wichtige Rolle. Freilebende Tiere etwa sind konstitutiv für gesellschaftliche Vorstellungen von naturbelassenen Landschaften und dem ländlichen Idyll (Macnaghten und Urry 1999; Jones 2003). Für Repräsentationen von Wildnis in Verbindung mit Namibia sind die big five (Elefant, Nashorn, Büffel, Löwe und Leopard) als flagship species ikonisch. Dass auch Wildpferde konstitutiv für gesellschaftliche Vorstellungen von Wildnis sein können und dass diese diskursiv umkämpft sind, zeigt Pütz (2017) am Beispiel der Ressourcenkonflikte um das Weideland der Mustangs in den USA. Diese werden v. a. entlang der Frage geführt, ob Mustangs überhaupt ,Wildpferde“ sind oder nicht vielmehr ,,verwilderte Pferde“. Dahinter steht die für die Praxis entscheidende Frage, ob sie als Bestandteil von ortsgebundener „Natur“ anzusehen sind, die schützenswert ist, oder als invasive Art, welche das Ökosystem, v. a. aber die wirtschaftlichen Interessen der Rinderfarmer bedroht.

Die Auseinandersetzungen um die Namib-Wildpferde schließen hier an, sind aber stärker im ökologischen Nachhaltigkeitsdiskurs und damit verbundener Praxis angesiedelt: Welche Tierarten ,gehören“ zu Namibias Naturlandschaft und haben daher unter der Leitlinie nachhaltiger Entwicklung besondere Schutzwürdigkeit? Anders formuliert: Welche Tiere haben im Kampf um die größte symbolische Bedeutung den Vorzug und „dürfen“ Namibias Naturlandschaft repräsentieren? Und das heißt letztlich auch: Welche Tiere dürfen überleben und welche nicht?

Anhand unserer Feldarbeiten können wir im Konflikt um den Umgang mit den Wildpferden drei rivalisierende Programmatiken identifizieren. Sie korrespondieren mit unterschiedlichen gesellschaftlichen Naturvorstellungen im Sinne von Mace (2014), sie ringen miteinander als Konzepte im Kampf um die nachhaltige Entwicklung der namibischen Nationalparks und sie erweisen sich als Frage von Leben und Tod für die Wildpferde und andere in der Region lebende Spezies. 
Eine erste Position propagiert Rahmensetzungen, die der „Natur ihren Lauf lassen“. Hierin zeigt sich ein Verständnis von Natur als frei von menschlichem Eingriff. Gebiete von Wildnis sind dementsprechend möglichst sich selbst zu überlassen und nur so „nachhaltig“ zu bewahren (nature for itself). Durch den Menschen eingeführte und am Leben erhaltene Pferde können demnach keine Wildpferde sein. Mehr noch: Jede Praxis zu ihrem Schutz gefährdet die ökologische Nachhaltigkeit „echter“ Natur. Dass eine wie auch immer gemanagte Natur bereits eine gesellschaftlich hergestellte Natur ist, wird dabei oftmals ausgeblendet. Der unterliegende Naturbegriff ist ein essentialistischer, streckenweise wird Natur zu etwas Göttlichem:

„Ich denke, manchmal machen Leute den Fehler, emotional zu werden über ein environmental issue. (...) Ich weiß, die Hyänen, die jagen. Die sind nicht nur scavenger [Aasfresser]. Aber (...) man kann sich nicht in die Trockenheit einmischen. Es ist act of god. Du kannst es nicht ändern. The survival of the fittest. “ (Wildparkmanager 2017)

Mit Naturkonstruktionen verbinden sich spezifische Anforderungen an die Praxis von Nachhaltigkeit. So erfordert die Auffassung nature for itself eine Festlegung, welche Tiere zur ortstypischen Natur gehören und welche nicht. In der Logik der Argumentation wird dies über die Dauer der Ansässigkeit gelöst, demnach waren die Hyänen ,zuerst da“ und haben mehr Recht auf Überleben als die vom Menschen eingeführten Pferde:

„And the MET also said, which we understand, that they are an indigenous species and the horses are not. So, if one has to stay and one has to go, the hyenas should stay and the horses should go. Because the hyenas belong there, it was always their territory, even if they haven't been there in big numbers and even if they haven\&apos; $t$ been so 'resident' as we call it. " (touristischer Anbieter 2017)

Eine zweite Position identifiziert die Hyänen als Schlüssel zur Lösung und schlägt vor, diese umzuziehen oder zu füttern und damit vom Erlegen der Pferde abzuhalten. Nachhaltigkeit ist aus dieser Perspektive nur (noch) durch menschlichen Eingriff zu sichern, der ein aus den Fugen gekommenes Gleichgewicht durch Intervention kurzfristig wiederherzustellen und langfristig $\mathrm{zu}$ erhalten vermag. Wenn die Hyänen also zum Problem für den Fortbestand für die Pferde werden, dann ist es angezeigt, einzugreifen:

„Since the situation developed as it did over the last ten years, of the hyenas increasing there and coming here and now have established quite a nice little setup here at Garub. 
This situation is not sustainablewith the horses. So they\&apos;ve killed all the foals for the last five years. " (Wildtierbiologin 2017)

Der unterliegende Naturbegriff zeigt sich als nostalgisch-arkadischer Zustand, in dem Mensch und Tier friedlich koexistieren, der Mensch aber dennoch verantwortlich für das Überleben von bestimmten Tieren (hier: Pferden) ist. Diese Figur des Menschen als Hirten verbindet sich mit der Rahmung von Naturschutz im Sinne von nature and people.

Eine dritte Position schlägt vor, die Pferde umzuziehen und vor den Hyänen ,in Sicherheit“" zu bringen:

„Da haben wir vorgeschlagen: Wir möchten diese Wildpferde nach Klein-Aus Vista nehmen [Flächen eines touristischen Anbieters]. Das ist der einzige Farmer, der bereit ist zu sagen, , die kriegen dort ihr Reich'. Und wir [die NWHF] schützen sie, diesen kleinen Kern, der noch übrig ist. “ (NWHF Aktivist 2017)

„And we think it would be good if it\&apos;s as close as possible to the area where they are now because that is kind of the same environment and habitat and it still remains as a tourism attraction in the area. Because it is really important for all of us. If you would remove the horses completely, for Aus tourism it would really be a big, big blow. " (touristischer Anbieter 2017)

Diese Position macht argumentativ nicht nur die Tiere selbst, sondern auch deren Nutzen und Nutzbarkeit zum Gegenstand. Nachhaltig ist nicht nur Biodiversität und Artenschutz, also die Sicherung des (genetischen) Überlebens der Pferde, sondern auch das Überleben der lokalen touristischen Ökonomie, die von den Pferden als touristisch nutzbare Konsumgüter (Pütz und Poerting 2020) abhängig ist. Interessant ist die Widersprüchlichkeit, die durch diese Verknüpfung aufbricht, denn sie erschwert eine eindeutige diskursive Zuordnung der Wildpferde. So kann touristischer Wert nur erreicht werden, wenn die Tiere sowohl als ,wild“ eingeordnet werden können als auch emotionale Verbundenheit zulassen. Verbundenheit mit Pferden wie auch die Sorge um sie sind ebenfalls durch mediale Diskurse - von Kinderfilmen über Jugendbücher bis hin zu künstlerischen Darstellungen und Visualisierungen in sozialen Medien (Poerting und Schlottmann 2020) - mächtige Repräsentationen von z. B. Hengsten (vgl. Abb. 3) oder aber Pferd-Mensch-Beziehungen, die sich dann in der touristischen Praxis des Fotografierens oder aber des Fütterns manifestiert. Dies konterkariert wiederum die 


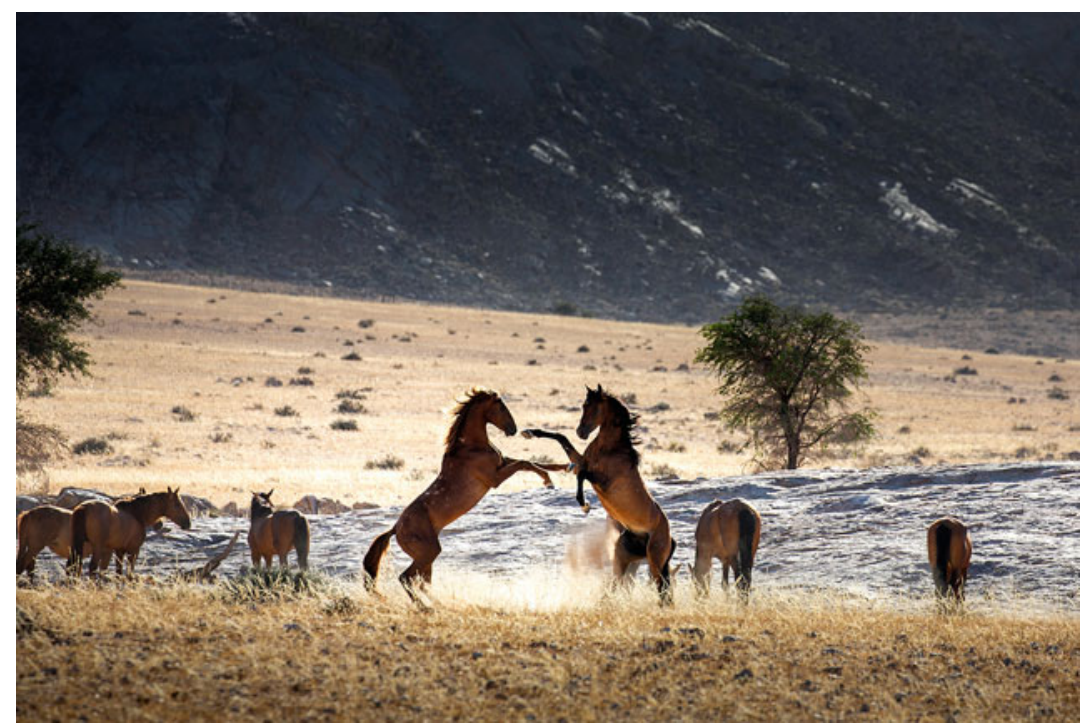

Abb. 3 Steigende Hengste bei Garub (2018, Photo: Teagan Cunniffe). Steigende Hengste sind seit der Antike durch zahllose Darstellungen gefestigt ein mächtiges Symbol und Visiotyp (Pörksen 1997) für Unbändigkeit und Wildheit. In der Namib begeben sich Touristen mit Kameras bewaffnet auf der Suche nach diesem Motiv; als Beleg für ihre Wildniserfahrung

Zuordnung der Tiere zu Wildnis als dem Menschen entgegengesetztes Natürliches, Ungezähmtes. ${ }^{2}$ Der inhärente Widerspruch ist indes geradezu konstitutiv für den ökonomischen Wert der Tiere. Sie müssen beides repräsentieren, müssen ,sowohl als auch“, wild und zahm, sein und noch dazu gut aussehen. Das Paradox entspringt einer anthropozentrischen Perspektive der Inwertsetzung von Natur (nature for people) und begründet, warum die Protagonisten damit ringen, Wildheit bewahren $\mathrm{zu}$ wollen und dadurch das Wilde gleichsam unmöglich zu machen:

„There is a couple paradox between 'wanting them wild' - and they must run around and they must show and beautiful scenery and so, and the stallions must kick and scream and all that [Abb. 3]. But on the other hand, the people find it really nice,

\footnotetext{
${ }^{2}$ Mit Schlünder (2012, S. 325) ließe sich auch sagen: Der Hunger von Pferden wird mit dem „Hunger“ von Menschen nach emotionaler Befriedigung, verbunden mit Akten der Zähmung und Sorge, synchronisiert.
} 
when the horses come up and eat out of the hand. Same person! And (...) who would really cry out loud when the horses look like they look now: skinny and scruffy." (Wildtierbiologin 2017)

Welche der Vorstellungen von Natur und zu schützender Wildnis sich als konkrete Ausgestaltung von ,nachhaltiger Praxis“ durchsetzt, erweist sich für die betroffenen Tiere allerdings als Frage von Leben und Tod. Sie können je nach Perspektive ,richtige“ Repräsentanten ortsgebundener Natur darstellen bzw. zum „richtigen“ tierischen Inventar des Wildparks gehören und überleben. Sie können als Invasoren wahrgenommen werden und werden (voraussichtlich) sterben.

Die ethische Frage nach dem Recht auf Überleben betrifft nicht zuletzt auch die wissenschaftliche Praxis als Teil des Diskurses zu Natur und ihrem Schutz. Wie ist sie positioniert und inwiefern gelingt es ihr, sich in der Rolle einer beobachtenden Instanz reflexiv zu distanzieren? In dieser Hinsicht werden Konzeptionen von nachhaltigem Naturschutz, welche die Bedürfnisse nicht-menschlicher Akteure wie vor allem von Tieren (gemeinhin unter „Natur“ subsummiert) vernachlässigen, jüngst vermehrt der Kritik des Anthropozentrismus ausgesetzt (Lorimer 2015, S. 15, s.a. Urbanik 2012, S. 17). Auf die damit verbundene erkenntnistheoretische Problematik kommen wir noch zurück. Wie unser Fall zeigen wird, kann ein Verständnis der Konflikte um nachhaltigen Naturschutz und Mensch-Tier-Interaktionen mit rein diskursanalytischen Verfahren jedoch nicht angemessen gelingen, weil dabei Aspekte der Materialität, der Körperlichkeit und einer - wie auch immer zu fassenden - agency of nature in einem ,Schreiben über" und damit außerhalb von Natur (Wolch 2002, S. 730) ausgeblendet werden.

\section{$4 \quad$ Nachhaltigkeit und Territorialisierung}

Alle dargestellten Positionen verbindet trotz der aufgezeigten Differenzen ein mehr oder weniger starkes Festhalten an dualistischen Natur-KulturVerständnissen. Das Denken in Kategorien von Nachhaltigkeit und die Ausrichtung des Handelns daran scheint - zumindest im Feld des Naturschutzes - unweigerlich verbunden mit einer Reproduktion der Trennung von Natur und Kultur. Unser Beispiel zeigt aber auch, dass diese diskursive Praxis der ontologischen Abgrenzung menschlicher und nicht-menschlicher Bereiche mit einer Praxis der Territorialisierung verbunden ist, die immer wieder neue Formen annimmt (Peluso und Lund 2011). Sie verräumlicht die Trennung zwischen Natur und Kultur auf unterschiedliche Weise und versucht, „Wildnis“ dauerhaft ihren spezifischen Ort zuzuweisen. Ihre materielle Dimension zeigt sich dabei am 
eindrücklichsten in Form des Zauns, der den Park begrenzt oder Gehege, Besucherbereiche u. ä. trennt. Dabei ist für uns grundlegend, dass räumliche Grenzen - materielle ebenso wie administrative oder eigentumsrechtliche, klassifikatorische ebenso wie semantische - gleichermaßen ermöglichen wie beschränken. Sie ermöglichen die Festschreibung, Strukturierung und Regelung gesellschaftlicher Naturverhältnisse und dabei erlauben sie insbesondere, Zugehörigkeiten und Verfügungsrechte festzulegen. Sie bestimmen, welches Tier wo Recht auf Schutz genießt, aber auch, wem die Tiere gehören und wer dementsprechend an ihrer Inwertsetzung in Form von Nutzung (auch touristisches Erleben) oder auch Tötung beteiligt sein darf. Nicht zuletzt beschränken sie auch Zugänge, Verfügbarkeiten und die Mobilität von Tier und Mensch und damit ihre erlebten und gelebten Räume.

\subsection{Nachhaltige Verortung von Natur}

Der Blick auf die im umkämpften Terrain der Nachhaltigkeit eingelagerten Praktiken der Territorialisierung offenbart die Funktionalität räumlicher Konzepte für die raumzeitliche Fixierung gesellschaftlicher Naturverhältnisse. Die mit einem Containerraumkonzept einhergehende essentialisierende Verortung von Lebewesen und Sachverhalten scheint einerseits ein konstitutives Element von Nachhaltigkeit, das Erhalten eines Zustands, zu unterstützen und vielleicht auch erst möglich zu machen: Räumliche Territorialisierung mit dem Ziel zeitlicher Fixierung. Der genaue Blick macht aber andererseits auch Widersprüche und Verwerfungen solcher Territorialisierungen sichtbar, die eher darauf hinweisen, dass es lohnend sein könnte, anstatt weiterhin die genaue Bestimmung von Natur (und Kultur), ihrer Territorien oder dort geltenden Regeln zu verhandeln, vielmehr die etablierten und institutionalisierten diskursiven wie materiellen Praktiken gesellschaftlicher Naturverhältnisse grundlegend anders zu konzipieren und in der Folge auch anders zu praktizieren.

Die Territorialisierungspraxis des MET fokussiert primär auf die Grenzen des Nationalparks. Sie produziert Widersprüchlichkeiten, da - wie gezeigt - Wildpferde nach MET nicht zur ,ursprünglichen“ ortstypischen Natur zählen (also zum „Außen“ gehören), auf der anderen Seite aber im Inneren der Parkgrenzen leben. Dies führt zur paradoxen Situation, dass das MET der von der NWHF vorgeschlagenen Umsiedlung und damit einhergehenden „Privatisierung“ der Wildpferde nicht zustimmen kann, obwohl es sie selber nicht als schützenswert erachtet: 
„Die sind Teil von unserem Park. Die kann ich nicht einfach rausholen und auf eine Farm bringen. " (Wildparkmanager 2017)

Eigentumsrechtliche Territorialisierungen sind mehr als profan, weil sie auf die grundlegendere Frage verweisen, ob und inwiefern ein von jemandem besessenes Tier, eines, das also diskursiv mehr als Eigentum denn als Tier angerufen wird, grundsätzlich überhaupt noch als ein wildes Tier gelten kann, und - in Bezug auf den Fall der Pferde -, inwiefern deren Leben auf Park- oder Farmgelände an dieser Frage etwas ändert.

Ziele der Wildtierbiologin sind hingegen die nachhaltige Bewahrung der ,natürlichen Lebensweise" der Pferde und ihr Überleben als Rasse. Auch dies stellt spezifische Anforderungen an Grenzziehungen. Ihre Forderung nach territorialer Integrität erfolgt aus Sicht der Pferde und deren räumlicher Praxis und lehnt dazu quer liegende Parkgrenzen grundsätzlich ab. Sie verknüpft dabei Argumente sozialer Nachhaltigkeit - Pferde repräsentieren koloniale Vergangenheit und sind damit „,kulturelles Erbe“ - mit Argumenten biologischer Nachhaltigkeit, insbesondere mit dem Erhalt von Biodiversität, da die Pferde über die Jahrzehnte auch genetisch zu einer eigenständigen Rasse geworden seien. Territorium, genauer, wo gelebt und wo gestorben wird, ist hierfür konstitutiv:

„And the thing is also, it is not so easy to just take the horses away and sell them off and so. Because then, first of all you lose the heritage. And you lose the genetic peculiarities. Plus, they also don\&apos; t do so well in other areas. (...) Then they can rather be hyena food then - that\&apos; s better for them “. (Wildtierbiologin 2017)

Hier wird deutlich, dass die Grenze des Nationalparks in Anlehnung an Foucault auch als eine biopolitische Grenze, eine Grenze von Biomacht aufgefasst werden kann (vgl. Beiträge in Chrulew und Wadiwel 2017). Diese wird insbesondere auch für die Hyänen relevant, die auf Privatland erschossen werden dürfen (und erschossen werden), in den Grenzen der Nationalparks aber nicht.

\subsection{Zäune}

Die offenbarste Materialisierung bzw. materielle Sicherung menschgemachter territorialer Grenzziehungen findet sich in unserem Beispiel in Form des Zauns. Dessen Ambivalenz liegt in seinem ermöglichenden und einschränkenden Charakter, im inklusiven Schutz von etwas Erhaltenswertem bei gleichzeitiger Exklusion 
von etwas Bedrohlichem. Das macht den Zaun weltweit zum bevorzugten Mittel von Naturschutz. Mit dem Umzäunen wird immer auch eine Trennung von Natur und Kultur reproduziert und definiert, was oder wer zugehöriges „Wildes“ oder fremder „Wilderer“ ist (Brockington 2002). Mit dem Ziel der Nachhaltigkeit errichtete Zäune versuchen, „Natur“ vor menschlichem Eingriff, vor invasiven Arten, Krankheiten und anderen Bedrohungen zu schützen. Zugleich regulieren sie den Zugang bestimmter Personen (i. d. R. Touristen) zu den Tieren (Evans und Adams 2016, S. 216, zu Zäunen als Technologie des Trennens auch Poerting et al. 2020).

Die Ambivalenz des Zaunes widerspiegelt somit die Ambivalenz von Natur als schützenswert (innen) und bedrohlich (außen). Im Falle Namibias stellt sich die Frage, ob die Wildpferde zur lokalen Artenvielfalt (innen) als bewahrenswert zählen oder als ,,invasive Art“ (außen) und für das lokale Ökosystem bedrohlich angesehen werden. Diese Perspektive erweist sich jedoch nicht nur biopolitisch als zu kurz greifend (Hinchliffe et al. 2013). Nimmt man die Perspektive der Wildpferde ein, verkehrt sie sich geradezu, leben die bedrohlichen Hyänen im Inneren, während ihnen der Zaun die Flucht ins sicherere Außen verwehrt.

Als materielles Hindernis für die Mobilität von Tieren, welche sich der menschlichen Praxis der Territorialisierung widersetzen, aber auch beugen müssen, zeigt der Zaun seine für ökologische Nachhaltigkeit widersprüchliche Wirkung:

„Und dann in den 80er Jahren hat man den Zaun gezogen. Dieser Zaun vom Staat, der die Wüste und das Farmland richtig geteilt hat. Weil da viel Wilderei war. Der Zaun hat natürlich viel Schaden angerichtet, da das Wild nicht mehr richtig hin und her ziehen konnte. Mehr Schaden als das, was die Farmer eigentlich gewildert oder geschossen haben. Und dadurch waren natürlich die Pferde jetzt eingeengt auf diesem Gebiet. Die konnten nicht mehr rein ins Townland nach Aus oder auf die Farm gehen. " (NWHF Aktivist 2017)

Widerständigkeit gegen die gesellschaftliche Verortung von Natur in Form ihrer symbolischen und materiellen Territorialisierungen zeigt sich aber auch in der Folge von Witterungseinflüssen sowie von Wasservorkommen und -verfügbarkeit. Deren Dynamik und Unsicherheit steht einer dauerhaften Fixierung eines (richtigen) Ortes von Wildnis genauso gegenüber, wie die Beweglichkeit der Pferde selbst:

„Also wenn man davon spricht: Man muss ein riesiges Gebiet kreieren, wo aber auch Freiräume sind von Ost nach West. Weil der Regen kommt immer vom Westen her oder von Osten her. Und der wenigste Niederschlag ist im Westen. Da wo die wilden 
Pferde jetzt sind. $10 \mathrm{~mm}$ dort jetzt. Geht man $30 \mathrm{~km}$ weiter, waren es schon $60 \mathrm{~mm}$. Die Chance, dass sie dort überleben können. Und diese Route ist jetzt nicht mehr da. “ (NWHF Aktivist 2017)

Während der Zaun also aus einer Perspektive des Parkmanagements der räumlichen Festschreibung einer bestimmten Natur und damit auch deren nachhaltiger Konservierung dienen soll, beschneidet er die räumliche Mobilität der Tiere und unterstützt gerade nicht die nachhaltige Sicherung des Pferdebestands, wie sie, wenn auch sehr unterschiedlich motiviert, von den zivilgesellschaftlichen Akteuren im Feld angestrebt wird.

\section{$5 \quad$ Perspektiven ontologischer Grenzüberschreitung}

Unser Beispiel macht die Problematik der gesellschaftlichen Institutionalisierung und Verortung einer Trennung von Natur und Kultur erkennbar, die durch räumlich gefasste Nachhaltigkeitspolitiken permanent erneuert wird und dabei fortwährend Widersprüchlichkeiten produziert. Gleichzeitig deutet sich bereits an, dass eine rein diskursanalytisch angelegte Betrachtung der Zusammenhänge zu kurz greift. In den letzten Jahren sind in der Humangeografie und verwandten Feldern verschiedene Überlegungen entstanden, wie der Natur-Kultur-Dualismus konzeptionell überwunden werden kann (Steiner 2014; Schröder und Steiner 2020). Diese sind zu großen Teilen inspiriert von nicht-repräsentationalen, posthumanistischen oder netzwerkorientierten Ansätzen (Hybridität, ANT, Assemblage). In Anschluss daran fordert z. B. Lorimer (2015, S. 5) eine ,neue Ontologie“, die auch konkrete Nachhaltigkeitspraxis anleiten und sich grundsätzlich von Vorstellungen einer zu bewahrenden Natur und den damit verbundenen Einschreibungen von Natur-Kultur-Differenzen lösen solle. Stattdessen schlägt er eine ,multinatürliche Ontologie von Wildnis“ (ebd.: S. 32) als Basiskonzept vor. Dieses akzeptiere Welt von vornherein als hybrid, als gleichermaßen konstituiert in Assemblagen von Menschen und Nicht-Menschlichem (Tiere, Technologien, Diskurse, Materialitäten) und als multipel in ihren raumzeitlichen Dynamiken.

\subsection{Assemblage und Hybridität}

Netzwerkorientierte Ansätze akzeptieren Hybridität als Kernbestandteil einer neuen Ontologie, in welcher sich Unterscheidungen zwischen Natur und Gesellschaft, Mensch und Tier, Organismen und Maschinen auflösen. So können in 
Anlehnung an Whatmore und Thorne $(1998,2000)$ Mensch-Wildtier-Beziehungen als Assemblagen konzipiert werden, die sich aus Nahrung, Raubtieren, Menschen, Institutionen, Daten, Algorithmen, Diskursen und Materialitäten zusammensetzen, als networks of human-animal relations (1998, S. 436 ff.). Auch die wildlebenden Namib-Pferde können somit als eingebettet in eine Assemblage betrachtet werden, welche die enthaltenen Elemente wie auch die Landschaft der Namib-Wüste ko-konstituiert.

Aus dieser Perspektive sind die Wildpferde - wie alle nicht-menschlichen Entitäten der Namib-Wüste - ausgestattet mit agency, d. h. mit Wirkmächtigkeit in Beziehung zu anderem und anderen. Sie widersetzen sich menschlichen Praktiken und fordern in spezifischen Situationen Handlungen heraus. Solchermaßen gefasst interagieren sie aktiv mit nicht-menschlichen Organismen (Hyänen, anderen Pferden, Gras etc.) sowie mit menschlichen Organismen (fütternden und gebissenen Touristen, pflegenden und zählenden Tierschützern, Hoteliers, Wildtierbiologen oder Rangern). Ihr Leben und Sterben bildet den Zweck von NGOs und Ressorts von Behörden, es erzeugt und stabilisiert ein Netzwerk unterschiedlicher Akteure und Organisationen, die sich mit ihm befassen.

Die Interaktionen der Wildpferde sind aber auch - wie gezeigt - eingebettet in umkämpfte Naturschutzdiskurse und Auslegungen von Natur, Wildnis und Nachhaltigkeit, in machtvolle Imaginationen des „Wildpferdes“, die durch Medien (z. B. Dokumentationen, Spielfilme) (re-)produziert werden. Auch sie sind Teil der Assemblage. Im speziellen Falle Namibias sind die Pferde darüber hinaus Gegenstand postkolonialer Diskurse, in denen Praktiken des Wildparkmanagements und Maßnahmen zum Schutz der Pferde aus ihrem kolonialen „Ursprung“ abgeleitet werden:

„Als ich mit dem Vorschlag kam, ,können wir vielleicht die Hyänen relocaten'? (...) Da war die Antwort: ,Kommt auf keinen Fall in Frage. Nur weil da so ein paar Deutsche sich die Pferde angucken möchten, müssen wir nicht gleich hier jetzt die Hyänen relocaten“". (NWHF Aktivist 2017).

„Das ist in unserer Umfrage, wo wir drei-vierhundert Responses hatten. Waren vielleicht ein oder zwei schwarzsprechende Namibier, Schwarze, die in diese Richtung gedacht haben: ,Die Pferde wurden gebraucht durch die Soldaten, um meine Vorfahren zu vernichten: " (NWHF Aktivist 2017)

Zudem erweisen sich kalkulative Verfahren (auch) in unserem Beispiel als wesentliches Element von Mensch-Wildpferd-Assemblagen: Die Tiere der Wildpferdeherden werden gezählt, beobachtet und in ihrer Mobilität kontrolliert. Der 
konkrete Umgang mit ihnen wird durch Algorithmen der Tragfähigkeit auf Basis hydrologischer Messreihen oder Bestockungsziffern bestimmt:

„Die carrying capacity wird ausgearbeitet bei uns in jedem Park. Wir wissen schon, wir haben Ranger, die gehen rein und sagen , large stock unit ..., small stock unit .... . “ (Wildparkmanager 2017)

Mischkalkulationen aus Fütterungskosten und diesen gegenübergestellte Spendenvolumina lösen Kampagnen der Öffentlichkeitsarbeit der NWHF aus, wodurch die Lebensbedingungen der Wildpferde Gegenstand (internationaler) Berichterstattung werden. Investitionen lokaler touristischer Anbieter in ihr Überleben werden mit erwarteten Einnahmeverlusten infolge ihres Aussterbens abgewogen. Kalkulationen zum erwarteten Aussterben schließlich lösen Fütterprogramme und biopolitische Maßnahmen an anderen Orten Namibias aus:

\begin{abstract}
„Nochmal zu den Hyänen. Das ist keine langfristige Lösung. Aber wir haben jetzt einfach jeden dritten Tag eine Stute weniger. Da kann man das Ende absehen. Und das Ende ist eben in 18 Monaten spätestens. (...) Wir wissen wo ihr Bau [der Hyänen] ist. Und dorthin liefern wir jetzt eben Rinder oder Pferde, die erlöst werden mussten, kranke Pferde und solche Sachen. Wir kaufen hauptsächlich Wild auch an. Wir haben so einen Farmer, der eine Überbevölkerung von Oryxen hat. Der liefert uns dann regelmäßig ein Oryx eben aus. Es gibt ja auch viele, die Wildfarmen haben, die Wild produzieren, sozusagen. Die dann, wenn es zu Trockenzeiten kommt, dann müssen die die Zahlen auch runterbringen. " (NWHF Aktivist 2017)
\end{abstract}

Schließlich ist die Interaktion der Wildpferde stark durch materielle Artefakte vermittelt. Neben dem bereits diskutierten Zaun des Wildparks zählen hierzu auch Bohrlöcher, Tränken, Futterkrippen etc. welche die alltägliche räumliche Praxis der Pferde sowie das auf die Versorgung oder die (touristische) Beobachtung der Pferde ausgerichtete Handeln der Menschen beeinflussen. Wie alle anderen Elemente des Mensch-Wildpferde-Netzwerkes, sind damit auch materielle Artefakte an der Ko-Konstitution von Landschaften wie der Namib-Wüste beteiligt (vgl. Abb. 4).

Die Einnahme von Assemblage-Perspektiven würde die derzeit im Naturschutz dominierenden, territorialisierenden und dabei Natur-Kultur separierenden Nachhaltigkeitspraktiken erheblich herausfordern. Denn aus Assemblage-Perspektive ist eine klare Zuordnung von Wildpferden zu einer wie auch immer definierten Kategorie „Natur“ konzeptionell nicht haltbar - alleine schon, weil Assemblagen immer als zeitlich und räumlich situiert anzusehen sind. Damit sind Dynamik 


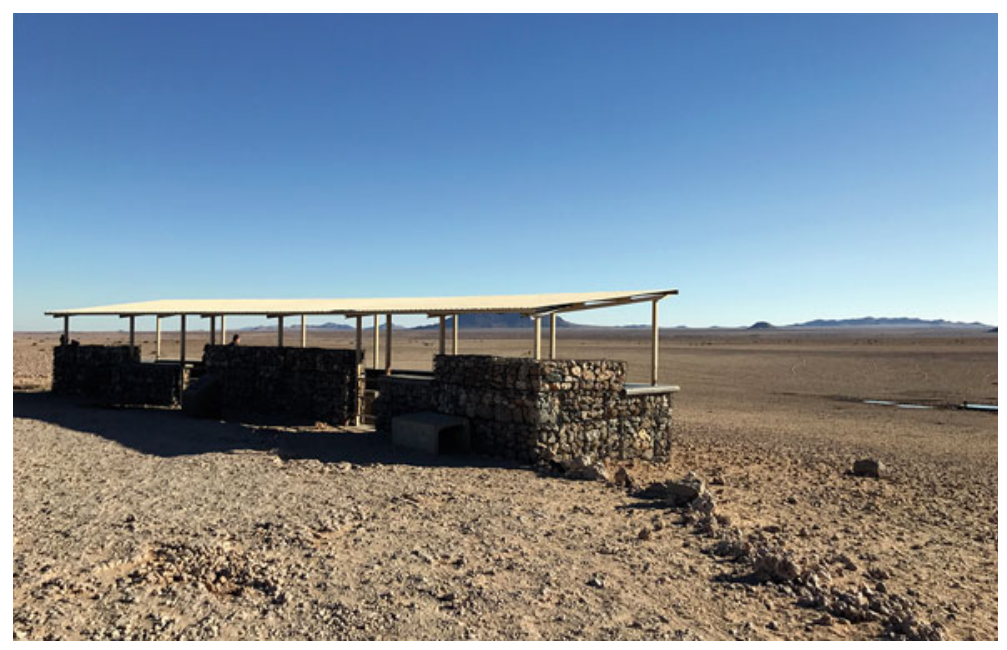

Abb. 4 Beobachtungsstation bei Garub. Ko-Konstitution von Wildnis in der ,naturalcultural contact zone“ (Haraway 2008): Halbwüste mit in Dürrezeiten quasi nicht vorhandenem Nahrungsangebot, bauliches Ensemble aus Tränke und Beobachtungsstation, das Körper der Pferde vor die Augen von Touristen zwingt, Immobilisierung der Pferde durch Tränken, infolgedessen landschaftsprägender Charakter der Pferdespuren. Tränken als Symbolisierung einer spezifischen Mensch-Pferd-Beziehung, bauliches Ensemble als Verweis auf spezifische Form internationalen Tourismus etc. (Foto: Pütz 2017)

und Wandel konstitutiv für Assemblagen und stehen dem bewahrenden Charakter von „Nachhaltigkeit“ diametral entgegen. Kategorisierungen von „Natur“ (und ihrem konstitutiven Anderen, der Kultur) sind hier weniger als ontologische Unterscheidungen relevant, sondern vielmehr in ihrer Funktion, z. B. für die Aufrechterhaltung oder Ablehnung bestehender Politiken. Durch diesen Fokus auf die Verwendung von Natur als Konzept lassen sich bedeutsame Zusammenhänge ihrer Ko-Konstitution erkennen, die Widersprüche und Reibungsflächen bestehender Nachhaltigkeitspraxis zum Vorschein bringen.

Darüber hinaus zeigen viele Beispiele unserer Untersuchung das Potenzial und die „Feld-Angemessenheit“ von Konzepten der Hybridität, welche Tiere als in zugleich biologisch wie sozial geformten Beziehungen konstituiert auffassen. Denn vor allem Pferde entziehen sich permanent dem Versuch einer eindeutigen Zuordnung zu Natur, nicht nur, weil - akzeptiert man sie ausgestattet mit agency - in ihrer Begegnung mit dem Menschen offensichtlich keine Grenze zwischen Natur und Gesellschaft bedeutsam ist, und nicht nur, weil sie - Lorimer (2015) 
folgend - über ein spezifisches ästhetisches, ökologisches und korporales „Charisma“ als biopower verfügen. Pferde symbolisieren wie wohl keine andere Spezies die menschliche Sehnsucht nach einer Verschmelzung von Natur und Kultur, wie sie paradigmatisch in der Figur des Zentauren zum Ausdruck kommt und seit Jahrtausenden Gegenstand künstlerischer Darstellungen ist. Dieser zutiefst hybride Charakter des Pferdes, the horse thing (s. u.), der auch durch die Jahrhunderte währende ,gemeinsame“ Unterwerfung der Welt erwuchs (erst Pferde ermöglichten dem Menschen die Überwindung räumlicher Distanz, physischer Grenzen, äußerer Feinde etc., vgl. z. B. Raulff 2015), äußert sich konkret in Widerständigkeiten gegen eine Zuordnung - sowohl diskursiv in der Figur des Pferdes als auch praktisch durch die direkte Erfahrung einer besonderen Emotionalität und, mit Merleau-Ponty (1966) gefasst, Interkorporalität:

„Because they do separate. Very few people I think can see them as wild animals. (...) If an oryx stands there with a broken leg they would say: 'Oh the oryx, shame'. But they would actually, at the end of the day, drive past. If it\&apos; s a horse, very often it\&apos;s a bit different. It\&apos;s the horse thing that comes in. " (Wildtierbiologin 2017)

\subsection{Interkorporalität}

Die Dimensionen „umkämpfter Nachhaltigkeit“ sind mit Blick auf Diskurse und Strategien nicht hinreichend erfasst. Assemblage-Perspektiven fügen insbesondere mit der Akzeptanz der handlungsleitenden Agency von nicht-menschlichen Organismen, materiellen Artefakten und kalkulativen Verfahren wesentliche Elemente hinzu und verweisen auf Hybridität als Konzept, in welchem sich Unterscheidungen zwischen Natur und Gesellschaft auflösen. Sie scheinen dabei aber - wie auch die vorherrschenden programmatischen Rationalitäten von Nachhaltigkeit - körperliche bzw. leibliche Aspekte situierter und konkreter Praxis und deren agency dennoch noch nicht hinreichend zu erfassen, wenn nicht gar auszublenden.

Aus dieser Ausblendung ergibt sich ein erheblicher blinder Fleck. Denn auch die Diskurse der Nachhaltigkeit mit dem Ziel der Bewahrung und Verdauerung eines gewollten ,natürlichen“ Zustands werden inkorporiert. Dies bezieht sich zum einen auf verinnerlichte gesellschaftliche Normen im Umgang mit Natur und ihrem Schutz, welche u. a. bestimmen, wie (welchen) Tieren entgegenzutreten ist. Zum anderen erleben aber Subjekte die Begegnung mit Wildpferden als leibliche und emotionale Erfahrung, die sie - insbesondere, wenn Entscheidungen 
um Leben und Tod anstehen - mit z. B. entgegenlaufenden Nachhaltigkeitsnormativen in Einklang bringen müssen. Dies erzeugt auf Ebene der Subjekte erhebliche Spannungen. Die wiederum ergeben sich im Wesentlichen aus Versuchen der Aufrechterhaltung einer Grenzziehung zu „Natur“.

So ringen die in den Konflikt um die Wildtiere eingebundenen Akteure permanent mit sich selber um die Frage, was noch „natürlich“ und was schon „,unnatürlicher" Einfluss ist, sie hinterfragen die Grenzziehungen und Grenzüberschreitungen, die sie in ihrer Alltagspraxis laufend vornehmen, und sie erleben die Widersprüchlichkeiten, die sich daraus ergeben. Dies zeigt zunächst eine verbale Reflexion der Fütterpolitik:

„Und jetzt sind wir in die Situation gekommen, wo wir uns vielleicht einen schwachen Moment erlaubt haben, die Hyänen auch noch zu füttern. Und jetzt füttern wir die Hyänen und wir füttern die Pferde. Und das ist natürlich kein idealer Zustand. " (NWHF Aktivist 2017)

Die konkrete situierte Praxis der Fütterung in der contact zone (Haraway 2008), die ja ein Moment intensivster Interaktion zwischen Mensch und Wildpferd ist, erweist sich als ein Moment leiblich erfahrener Interkorporalität (Abb. 5). Die Wildpferde kennen die Zeitpunkte und Orte der Fütterung. Sie halten sich dementsprechend zu bestimmten Zeiten dort auf und nehmen das Heu entgegen, das vom LKW geworfen wird. Das Füttern löst intensive Interaktionen unter den Pferden, mit den Pferden und mit den beteiligten materiellen Artefakten (Futter, LKW, Tröge ...) aus: Rangeleien um Futter und Rangordnung, Aufeinanderbeziehen von Körpern unter Pferden und zwischen Pferden und Menschen, Momente gestischen und leiblichen Aufforderns, Berührungen, Gerüche, Verletzungen. Dieser dance of encounters (Haraway 2008: S. 4) steht für Grenzüberschreitung par excellence. Die Wildtierbiologin reflektiert dies. Ihre Fütterungspraxis kann als ,inkorporierte Grenzarbeit" gelesen werden, als ein permanenter Versuch, eine natürliche Ordnung trotz Zufütterung (nachhaltig) aufrecht zu erhalten und damit dem Normativ zu entsprechen, die Wildpferde als essenziellen Bestandteil ortsgebundener Natur zu erhalten. Sie berührt die Pferde nicht aktiv, sie pflegt oder versorgt nicht ihre Verletzungen, sie versucht, ihre Interaktion auf ,wissenschaftliche“ Praktiken des Messens und des Beobachtens zu reduzieren, immer von dem Anspruch geleitet, die Grenze zu „Natur“ zu respektieren:

„When I started, it was to me: I\&apos;m not going to touch them. I\&apos;m not going to try to touch them. If they come to me I\&apos; $m$ not going to chase them away. So I keep a line. So I don\&apos;t make an effort to make friends with them. And I\&apos;ve 
always just kept that. So I-I mean, I love them as much as I love my domestic horses. But. I understand life and death. " (Wildtierbiologin 2017)

Die inkorporierte Grenzarbeit von Subjekten bedeutet damit immer auch Arbeit an den eigenen Emotionen. Im Falle der Wildtierbiologin steht sie für den Versuch einer Grenzziehung zwischen einer als emotional und körperlich akzeptierten Verbindung zum (eigenen) Hauspferd und einer eher von distanzierter wissenschaftlicher Beobachtung geprägten Beziehung zum Wildpferd. Letztere rekurriert auf eine vermeintliche Grenze zur Natur und schließt damit bestimmte Formen der Interaktion aus.

Wie die Fütterungspraxis zeigt jedoch auch die Auseinandersetzung mit der emotional-körperlichen Mensch-Pferd-Beziehung das Scheitern des Versuchs einer Aufrechterhaltung einer Grenze zur Natur:

A: „When you go through the years, where you see them dying, the draught, whatever you learned to be a bit more, callus, I would say. “ B: „But they still all have a name. “

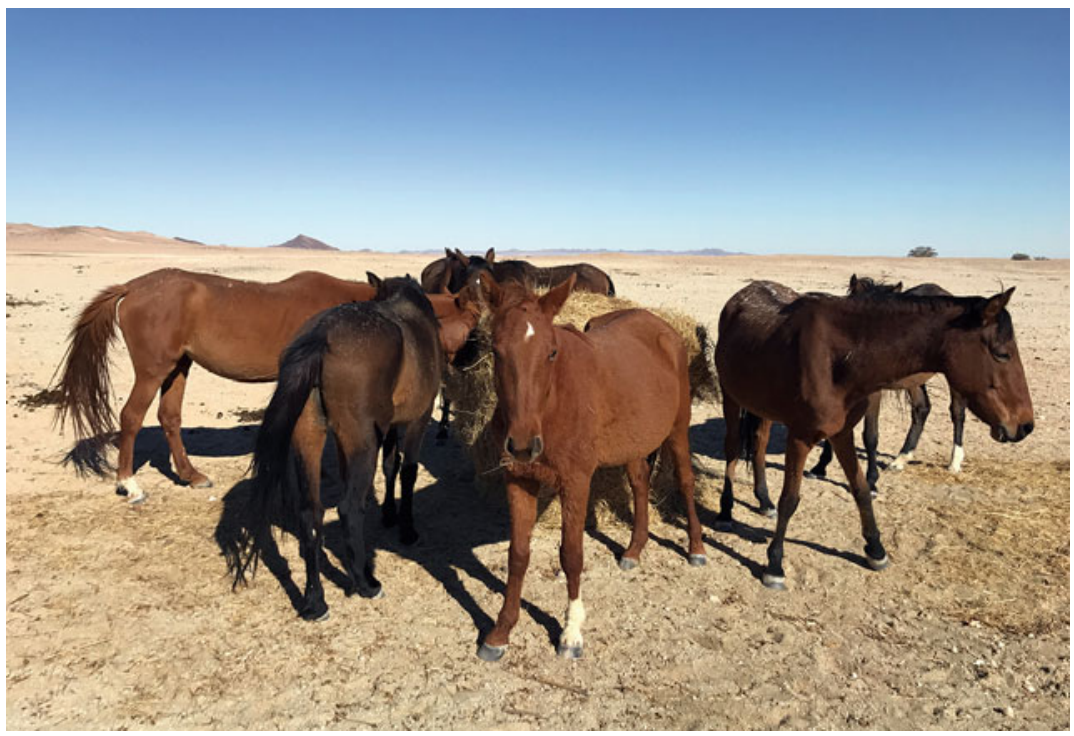

Abb. 5 „Ich lebe im Ausdruck des Anderen, und spüre, dass er in meinem lebt“ (MerleauPonty, 1964: S. 146). Ein Moment von Interkorporalität in einer Füttersituation. (2017, bei Garub, Foto: R. Pütz) 
A: „They still all have a name. Some just strike you a bit more. Yeah, obviously-there are certain individuals that I particularly like. And I really get cross if the hyenas catch those ones. But. It\&apos; s life. It\&apos; s the circle of life. That\&apos;s how it goes. “ B: „You kind of have to. “ A: „You have to. Yes. That\&apos;s how it is. That\&apos;s life. “ (NWHF Aktivisten 2017)

Alle Pferde haben einen Namen erhalten. Jedes einzelne ist der Biologin in seiner körperlichen Konstitution wie auch seinen charakterlichen Eigenheiten vertraut. Dieses Wissen ist generiert aus der leiblichen Erfahrung von Mensch-WildpferdBegegnungen und solchermaßen grenzüberschreitend. Es hat in den gängigen Rationalitäten von Nachhaltigkeit und Naturschutz jedoch keinen Platz.

\section{$6 \quad$ Eine neue Ontologie der Nachhaltigkeit?}

Nachhaltigkeit ist wissenschaftlich ein hoch problematischer Begriff, der durch seine Unterbestimmtheit analytisch kaum zu gebrauchen ist. Auch in der Praxis scheint es kein lohnendes Unterfangen, eine ,richtige“ Nachhaltigkeit zu verhandeln, solange die unterliegenden Verständnisse (z. B. Naturvorstellungen) unsichtbar bleiben, implizit (z. B. in Konflikten um die praktische Umsetzung) hochgradig umkämpft und durch ihre Unsichtbarkeit aber letztlich nicht verhandelbar sind.

Insofern ist unser Vorschlag für die wissenschaftliche Praxis zunächst, auf den Begriff der Nachhaltigkeit zu verzichten und stattdessen pragmatisch zu betrachten, worum genau es jeweils geht: Um eine langfristige Sicherung bestimmter Arten und wenn ja, welcher? Um Tierschutz und wenn ja, für wen? Um die Erhaltung von Natur und wenn ja, welcher und wer bestimmt diese mit welcher Rechtfertigung? Aus dieser Perspektive werden die im jeweiligen Kontext vorhandenen Widersprüche und Reibungsflächen fassbar und es entsteht grundlegende Erkenntnis über die Paradoxie eines Praxisfeldes, das seinen eigenen Begriffskern permanent konterkariert: Nachhaltigkeit, weil sie in ihrer Relationalität hoch umkämpft ist, kann nicht nachhaltig sein.

Unsere Betrachtung der Situation der Wildpferde in Namibia hat gezeigt, dass eine diskursanalytisch angelegte Untersuchung des Gebrauchs und der Durchsetzung von Nachhaltigkeit zwar wichtige Einsichten zu Deutungshoheiten und machtvollen Territorialisierungen erlaubt. Sie ist jedoch nicht hinreichend, um dieses Feld, das sich ja sowohl um die Fragen zukünftiger gesellschaftlicher Naturverhältnisse als aber auch um tägliche Entscheidungen des Lebens und 
des Leben-Lassens eröffnet, in seinen Widersprüchlichkeiten und deren Konsequenzen zu erfassen. Die Rolle von (territorialisierter) Materialität, deren Widerständigkeit und agency, verbunden mit verinnerlichten Normativen, körperlicher und leiblicher Interaktion und Emotionalität sind hierfür in die wissenschaftliche Betrachtung einzubeziehen.

Lorimer (2015, S. 5) plädiert für eine neue Ontologie von Natur unter dem Begriff „Wildnis“ (wildlife), den er von (klassischerweise mit Nachhaltigkeit verbundenen) Konzepten von Stabilisierung, Verdauerung und Gleichgewicht entkoppelt und stattdessen mit Konzepten von Dynamik, Prozesshaftigkeit und permanenter Dysbalance verbindet (ebd.: S. 7). In der Konsequenz gilt es ihm zufolge nicht nur, Praktiken der Verortung von Natur aufzugeben und Naturschutz als ein immer vorläufiges, herantastendes Verfahren anzusehen, sondern insbesondere auch, spezifisches Wissen zu akzeptieren und zu aktivieren, das aus einem learning to be affected hervorgeht, aus der leiblichen Praxis der Erfahrung von Mensch-Tier Begegnungen. Eine daraus hervorgehende Conservation after nature könnte helfen, manche der aufgezeigten Widersprüchlichkeiten zu überwinden (Lorimer 2015, S. 54):

\begin{abstract}
„Here, knowledge about the nonhuman world emerges out of situated, embodied, and technological encounters with the nonhumans that are the subject of research. The bodies of scientists are vital for this endeavor. It is only through training and experience that a scientist can learn to be affected by their target organism, ecology, or process. Technologies enhance and extend the possibilities of perception and recording. Science is propelled and guided by scientists' affective attachments to particular species and places. Habits and passions matter here and should be acknowledged, cultivated, and celebrated. There are multiple affective logics at work in conservation that shape what knowledge gets produced and what is accepted as a legitimate account. This is a multinatural approach, with the potential for difference and discord. Finally, natural knowledge is shaped by the relational agencies or biopower of nonhumans - in this case, in the form of nonhuman charisma."
\end{abstract}

Vor dem Hintergrund institutionalisierter Erkenntniskategorien in menschlichen Denktraditionen und Sprachgebrauch ist ein solches Unterfangen, auch dies hat unsere Betrachtung gezeigt, in der Tat eng an die kritische Auseinandersetzung mit in Wissenschaft und Alltagspraxis herrschenden ontologischen Imperativen und an die Entwicklung und mögliche Durchsetzung neuer, ,post-dualistischer“ Ontologien gekoppelt (Schlottmann et al. 2010). Denn territorialisierende Nachhaltigkeitspolitiken, paradigmatisch zu erkennen in Nationalparks wie dem Namib-Naukluft Park in Namibia, basieren grundlegend auf einer ontologischen Trennung von Natur und Kultur und diese ist Teil ihrer Wirklichkeit. Sie zeigt sich in tiefgreifenden Auseinandersetzungen, was zur (lokalen) Natur zählt, welche 
Tiere und Pflanzen sie repräsentieren (dürfen) und welche invasiv oder unrein sind und damit keine Schutzwürdigkeit genießen oder sogar - zum Schutz der ,authentischen" Lokalnatur - eliminiert werden müssen. Grenzen von Nationalparks und deren Materialisierung in Form von Zäunen sind - wie gezeigt wurde - Grundlage und Fixierung rechtlicher wie lebenspraktischer Regelung solch dualistischer Natur-Gesellschaft-Konstruktionen.

Auch dem aktuell viel kritisierten „Anthropozentrismus“ lässt sich nicht einfach entkommen, insofern er auf eine sozialisatorische Verinnerlichung des cartesianischen Dualismus verweist. Er ist tief verankerter Teil einer gewachsenen Wissenskultur und ihrer Kommunikation. So bleibt erstmal nur anzuregen, die wissenschaftliche Position zu verschieben, indem andere Fragen gestellt werden, die z. B. nicht auf den Sinn und Unsinn von bestimmten Praktiken im Mensch-Tier-Interaktions-Feld allein in Bezug auf den Menschen gerichtet sind (Urbanik 2012, S. 17). Das heißt dann nicht, anzunehmen, dass der Natur-Kultur Dualismus einfach zu überwinden ist, und auch nicht, dass er nicht kontextuell betrachtet funktionell ist. Er ist, wie seine Materialisierung der Zaun, Ermöglichung und Einschränkung. Doch diese „Ordnung der Dinge“ (Foucault 1974) ist nicht die einzige Möglichkeit des Verstehens von Welt. Sie ist demnach kritisch zu reflektieren, um neue Gestaltungsoptionen zu eruieren, um gesellschaftliche Naturverhältnisse zunächst verstehen und ggf. verändern zu können.

Lorimers Arbeiten, wie die anderer aus der Akteur-Netzwerk bzw. morethan-representational Perspektive argumentierender Wissenschaftler*innen sind in der Konsequenz zunächst einmal als Bemühungen um alternative Ontologien von Natur zu sehen, die ermöglichen, neben der diskursiven Dimension anderen, vor allem erfahrungsbezogenen Dimensionen der Naturbestimmung Einlass zu gewähren. An das herrschende Verständnis von Wissenschaft scheinen solche Bemühungen oftmals wenig anschlussfähig, wie schon Whatmores (2002) Vorschlag, Geographie als ein „Kunsthandwerk“ zu denken, gezeigt hat. Den Anschlussschwierigkeiten zugrunde liegt nicht zuletzt ein Verständnis von einer sich in ihren Gegenstand nicht einmischen dürfenden objektiven Wissenschaft. Was dabei selten zur Diskussion gebracht wird ist aber, dass wissenschaftliche Praxis sich unweigerlich einmischt und Position bezieht, wenn sie etwa einem bestimmten Natur- und - hier besonders hervorgehoben - Nachhaltigkeitsverständnis folgt und dabei implizit Position bezieht. Sie entscheidet dann mit, ob es um Bewahrung einer (z. B. durchweg positiv gesetzten) Artenvielfalt, der nationalen Wirtschaft, des Unterhalts eines Kleinbauern etc. und damit gleichsam nicht um Bewahrung von anderem, z. B. einer Herde von Wildpferden, geht. Und so sind Ansätze wie more-than representational, more-than human oder ANT auch 
als Bemühungen zu sehen, sich aus diesen wissenschaftstheoretischen Widersprüchen expliziter Objektivität und implizitem taking sides zu lösen, weil sie Kontingenz in hohem Maße erwarten und daher die Vorläufigkeit von Erkenntnis akzeptieren - und damit quasi vor der dann beobachtbaren Kategorisierung und Grenzziehung agieren. Eine solche Vorgehensweise wird umso plausibler, wenn vorausgesetzt wird, dass auch Wissen inkorporiert ist und eine leibliche Dimension hat und dass soziale Ordnung verinnerlicht ist (Jäger 2004).

Die gegenwärtige Rationalität von Nachhaltigkeit als Ziel von Naturschutz jedenfalls, das wurde deutlich, sieht Emotionalität und inkorporierte Erfahrung in Mensch-Wildtier-Begegnungen nicht vor. Sie abstrahiert vielmehr von der leiblichen Erfahrung von Menschen. Auch Assemblage-Ansätze zeigen sich hier eher blind. Insofern erscheint uns ein noch stärkerer Einbezug von Leiblichkeit in die wissenschaftliche Betrachtung von Mensch-Tier-Verhältnissen durchaus vielversprechend, will man die blinden Flecken wissenschaftlicher Beschäftigung mit gesellschaftlichen Naturverhältnissen verkleinern. Und hierzu lässt sich nicht nur auf neue Ansätze der emerging fields im ausgerufenen Anthropozän, etwa der environmental humanities oder animal geographies, zurückgreifen, sondern auch auf eine ganze Breite klassischer phänomenologischer Ansätze. Diese wären, ggf. neu gelesen, einerseits für Themen körperlicher Anrufung und Normierung, aber insbesondere auch von leiblicher Erfahrung in Mensch-Tier-Begegnungen fruchtbar zu machen, wie an anderer Stelle ausgeführt wurde (Pütz 2019a, 2021).

„Ich lebe im Gesichtsausdruck des anderen und fühle, wie er in meinem lebt“, schreibt etwa Merleau-Ponty im Rahmen seiner Entwicklungen des „Phänomenologie der Wahrnehmung“ (1966), und es ist insbesondere Merleau-Pontys Begriff der „Interkorporalität“, der uns mit Dutton (2012) als vielversprechender Ausgangspunkt erscheint (vgl. auch Whatmore 2002, S. 5). Interkorporalität beschreibt die leibliche Verbundenheit zur Welt und den begegnenden Anderen. Sie ist als leibliche Intersubjektivität nicht zwischen zwei Körpern, sondern wird als mit diesen (in der visuellen, haptischen etc. Begegnung) leiblich bereits verbunden entworfen (Haller 2017). So ließe sich auch in Anschluss an Lorimers ' Vorschlag um den Begriff wildlife ein um das Leibliche erweiterter Begriff der Wildnis erkenntnisleitend einbringen (Schlottmann 2019). Auch eine Weiterführung der (heuristischen) Unterscheidung von Körper und Leib erscheint uns fruchtbar für die Analyse von Mensch-Tier-Beziehungen, insofern mit ihr leiblichen Aspekten besondere Aufmerksamkeit geschenkt werden kann, sie aber auch in ihrer Widersprüchlichkeit etwa zu inkorporierten Normen und der diskursiven Konstruktion von menschlichen und tierischen Körpern offengelegt werden können. Diese Perspektiven einer ,neuen Tiergeographie“ (Pütz et al. 2021) führen u. a. auch zur wissenschaftlichen Analyse von Situationen, in denen die Grenzen 
zwischen Mensch und Tier sich verflüssigen, z. B. die Führung von Blindenhunden (Higgin 2012) oder Reiten (Pütz 2019b), eignen sich aber auch als Basis für eine andere Ontologie von Nachhaltigkeit, die derart aus Interaktionen gewonnenes Wissen akzeptiert. Learning to be affected, evtl. weitergehend gedacht auch learning to be intercorporeal, wäre dann jedenfalls nicht als widersprüchliche Praxis von Wildtierbiologen anzusehen, die permanent nicht nur die Grenze zwischen Natur und Gesellschaft infrage stellt. Solches Lernen von Zwischenleiblichkeit, als ein „mutual becoming and becoming horse“ (Spannring 2019) wäre als Praxis zu akzeptieren, welche die Grenze zwischen rationaler wissenschaftlicher Beobachtung (mit entsprechenden Technologien des Zählens, Messens und Kartierens) und empathischem Erleben aufhebt und damit auch die Basis für eine grundlegend andere wissenschaftliche Praxis bildet.

Danksagung Für anregende Diskussionen danken wir Kolleg*innen der Arbeitsgruppe „Geographien von Mensch-Tier-Verhältnissen“ - Olivier Graefe, Jürgen Hasse, Lisa Krieg, Julia Pörting, Verena Schröder und Christian Steiner - sowie Simone Pütz.

\section{Literatur}

Brand, K., und G. Jochum. 2000. Der Deutsche Diskurs zur nachhaltigen Entwicklung. Abschlußbericht eines DFG-Projekts zum Thema ,Sustainable Development/Nachhaltige Entwicklung - Zur sozialen Konstruktion globaler Handlungskonzepte im Umweltdiskurs “. München: MPS.

Brockington, D. 2002. Fortress conservation. The preservation of the Mkomazi Game Reserve Tanzania. Oxford: Currey.

Chrulew, M., and D. J. Wadiwel. 2017. Foucault and animals. Leiden: Brill.

Dutton, D. 2012. Being-with-animals. Modes of embodiment in human-animal encounters. In Crossing boundaries. Investigating human-animal relationships, Hrsg. L. Birke und J. Hockenhull, 69-85. Leiden: Brill.

Evans, L., und W. Adams. 2016. Fencing elephants. The hidden politics of wildlife fencing in Laikipia. Kenya. Land Use Policy 51:215-228.

Foucault, M. 1974. Die Ordnung der Dinge. Eine Archäologie der Humanwissenschaften. Frankfurt a. M.: Suhrkamp.

Goldbeck, M., T. Greyling, und R. Swilling. 2011. Wilde Pferde in der Namibwüste. Windhoek: Friends of the wild horses.

Haraway, D. 2003. The companion species manifesto. Dogs, people, and significant otherness. Chicago: Prickly Paradigm Press.

Haraway, D. 2008. When species meet. Minneapolis: University of Minnesota Press.

Haller, M. 2017. Interkorporalität. In Handbuch Körpersoziologie, Hrsg. G. Klein et al., Bd. 1, 45-49. Wiesbaden: Springer VS.

Higgin, M. 2012. Being guided by dogs. In Crossing boundaries. Investigating human-animal relationships, Hrsg. L. Birke et al., 73-90. Leiden: Brill. 
Hinchliffe, S., J. Allen, S. Lavau, N. Bingham, and S. Carter. 2013. Biosecurity and the topologies of infected life: From borderlines to borderlands. Transactions of the Institute of British Geographers 38 (4): 531-543.

Jäger, U. 2004. Der Körper, der Leib und die Soziologie. Entwurf einer Theorie der Inkorporierung. Königstein: Helmer

Jones, O. 2003. The restraint of beast': Rurality, animality, actor network theory and dwelling. In Country Visions, Hrsg. P. Cloke, 283-307. London:Harlow Pearson.

Laclau, E. 2002. Emanzipation und differenz. Wien: Turia \& Kant.

Lorimer, J. 2015. Wildlife in the Anthropocene. Conservation after nature. Minneapolis: University of Minnesota Press.

Mace, G. 2014. Whose conservation? Science 345 (6204): 1558-1560.

Macnaghten, P., and J. Urry. 1999. Contested natures. London: Sage.

Merleau-Ponty, M. 1964. The child's relations with others. In The primacy of perception, Hrsg. J. M. Edie, 96-155. Evanston IL: Northwestern Universities Press.

Merleau-Ponty, M. 1966. Phänomenologie der Wahrnehmung. Berlin: de Gruyter.

MET (Ministry of Environment and Tourism Namibia). 2013. Management Plan NamibNaukluft Park. Windhoek: Selbstverlag.

Peluso, N., and C. Lund. 2011. New frontiers of land control: Introduction. Journal of Peasant Studies 38 (4): 667-681.

Poerting, J., J. Verne, und L. Krieg. 2020. Gefährliche Begegnungen. Posthumanistische Ansätze in der technologischen Neuaushandlung des Zusammenlebens von Mensch und Wildtier. Geographische Zeitschrift 108 (2020) 3: 153-175.

Poerting, J., und A. Schlottmann. 2020. Das Charisma der Petfluencer: Zur Medialisierung konsumtiver Mensch-Tier Beziehungen am Beispiel Instagram. Berichte. Geographie und Landeskunde 93 (2020) 1-2: 145-170.

Pörksen, U. 1997. Weltmarkt der Bilder. Eine Philosophie der Visiotype. Stuttgart: Clett-Cotta.

Pütz, R. 2017. Wildpferde in den USA. Ressourcenkonflikte, Wildniskonstruktionen und Mensch-Wildtier-Verhältnisse. Geographische Rundschau 10:46-51.

Pütz, R. 2019a. Pferderücken. In Räume der Kindheit. Ein Glossar, Hrsg. J. Hasse und V. Schreiber, 259-265. Bielefeld: transcript.

Pütz, R. 2019b. Versuchen Sie, mit der Zartheit Ihrer Hilfen Neugier zu erwecken. Reiten als Zwischenleiblichkeit. Piaffe 24 (1): 54-59.

Pütz, R. 2021. Making companions: Companionability and encounter value in the marketization of the American Mustang. Environment and Planning E: Nature and Space 4(2): 585-602. https://doi.org/10.1177/2514848620924931.

Pütz, R., und J. Poerting. 2020. Mensch-Tier-Verhältnisse in der Konsumgesellschaft. Berichte. Geographie und Landeskunde 93 (1-2): 123-143.

Pütz, R., und A. Schlottmann. 2020. Contested conservation-neglected corporeality: The case of the Namib wild horses. Geographica Helvetica 75 (2020)2: 93-106 https://doi. org/10.5194/gh-75-93-2020.

Pütz, R., A. Schlottmann, und E. Kornherr. 2021. Einführung in die neue Tiergeographie. In Mehr-als-menschliche Geographien. Schlüsselkonzepte, Beziehungen und Methodiken, Hrsg. C. Steiner, V. Reiser, und G. Rainer. Stuttgart: Steiner (im Druck).

Raulff, U. 2015. Das letzte Jahrhundert der Pferde. München: Beck.

Schlottmann, A., O. Graefe, und B. Korf. 2010. Things that matter. A dialogue on interpretative and material semiotics in geography. Geographische Zeitschrift 98 (4): 226-236. 
Schlottmann, A. 2019. Wildnis. In Räume der Kindheit. Ein Glossar, Hrsg. J. Hasse und V. Schreiber, 378-384. Bielefeld: transcript.

Schlünder, M. 2012. Wissens-Hunger im Stall. Die Entstehung von Knochen-Schafen als Versuchstiere in der Unfallchirurgie. Berichte zur Wissenschaftsgeschichte 35 (4): $322-$ 340.

Schröder, V., und C. Steiner. 2020. Pragmatist Animal Geographies. Mensch-WolfTransaktionen in der schweizerischen Calanda-Region. Geographische Zeitschrift. https:// doi.org/10.25162/gz-2020-0003.

Schwartz, S. 2016. Nachhaltigkeit als Komplexitätsfalle. Grundmuster eines politischen Diskurses zwischen Hoffnung und Enttäuschung. Münster: Universitäts- und Landesbibliothek Münster.

Spannring, R. 2019. Mutual becomings? In search of an ethical pedagogic space in humanhorse-relationships: Interdisciplinary approaches to curriculum and pedagogy. In Animals in Environmental Education, Hrsg. T. Lloro Bidard und V. Banschbach, 79-95. Cham: Palgrave Macmillan.

Steiner, C. 2014. Pragmatismus - Umwelt - Raum. Stuttgart: Steiner.

Swilling, R. 2020. Running with the wild horses of the Namib. In Country life, https://www. countrylife.co.za/travel/heritage/wild-horses-namibia Zugegriffen: 09. Juni 2020.

Tremmel, J. 2003. Nachhaltigkeit als politische und analytische Kategorie. Der deutsche Diskurs um nachhaltige Entwicklung im Spiegel der Interessen der Akteure. München: Ökom-Verlag.

Urbanik, J. 2012. Placing animals. Lanham: Rowman \& Littlefield Publishers.

Whatmore, S. 2002. Hybrid geographies. London: Sage.

Whatmore, S., und L. Thorne. 1998. Wild(er)ness: Reconfiguring the geographies of wildlife. Transactions of the Institute of British Geographers 23 (4): 435-454.

Whatmore, S., und L. Thorne. 2000. Elephants on the move. Spatial formations of wildlife exchange. Environment and planning D: society and space 18 (2): 185-203.

Wolch, J. 2002. Anima urbis. Progress in Human Geography 26 (6): 721-742.

Pütz, Robert, Prof. Dr., Professur für Humangeographie am Institut für Humangeographie der Goethe-Universität Frankfurt am Main.

www.humangeographie.de/puetz

Schlottmann, Antje, Prof. Dr., Professur für Geographie und ihre Didaktik am Institut für Humangeographie der Goethe-Universität Frankfurt am Main.

www.humangeographie.de/schlottmann 
Open Access Dieses Kapitel wird unter der Creative Commons Namensnennung 4.0 International Lizenz (http://creativecommons.org/licenses/by/4.0/deed.de) veröffentlicht, welche die Nutzung, Vervielfältigung, Bearbeitung, Verbreitung und Wiedergabe in jeglichem Medium und Format erlaubt, sofern Sie den/die ursprünglichen Autor(en) und die Quelle ordnungsgemäß nennen, einen Link zur Creative Commons Lizenz beifügen und angeben, ob Änderungen vorgenommen wurden.

Die in diesem Kapitel enthaltenen Bilder und sonstiges Drittmaterial unterliegen ebenfalls der genannten Creative Commons Lizenz, sofern sich aus der Abbildungslegende nichts anderes ergibt. Sofern das betreffende Material nicht unter der genannten Creative Commons Lizenz steht und die betreffende Handlung nicht nach gesetzlichen Vorschriften erlaubt ist, ist für die oben aufgeführten Weiterverwendungen des Materials die Einwilligung des jeweiligen Rechteinhabers einzuholen. 